\title{
Kinetic equilibration in heavy ion collisions: The role of elastic processes
}

\author{
Julien Serreau \\ Institut für Theoretische Physik der Universität Heidelberg, \\ Philosophenweg 16, D-69120 Heidelberg, Germany \\ Dominique Schiff \\ Laboratoire de Physique Théorique, \\ Bâtiment 210, Université Paris-Sud, 91405 Orsay, France
}

(November 25, 2018)

\begin{abstract}
We study the kinetic equilibration of gluons produced in the very early stages of a high energy heavy ion collision in a "self-consistent" relaxation time approximation. We compare two scenarios describing the initial state of the gluon system, namely the saturation and the minijet scenarios, both at RHIC and LHC energies. We argue that, in order to characterize kinetic equilibration, it is relevant to test the isotropy of various observables. As a consequence, we find in particular that in both scenarios elastic processes are not sufficient for the system to reach kinetic equilibrium at RHIC energies. More generally, we show that, contrary to what is often assumed in the literature, elastic collisions alone are not sufficient to rapidly achieve kinetic equilibration. Because of longitudinal expansion at early times, the actual equilibration time is at least of the order of a few fermis.
\end{abstract}

PACS numbers: 25.75.-q, 12.38.Mh

\section{INTRODUCTION}

The main interest of studying very high energy heavyion collisions is the possibility that a quark-gluon plasma (QGP) be formed. It is widely believed that a large amount of real partons (essentially gluons) with transverse momentum $p_{t} \sim 1-2 \mathrm{GeV}$ is freed in the very early stages of such collisions, carrying most of the produced transverse energy [1,2]. While rapidly diluted by longitudinal expansion, this parton gas tends to locally equilibrate via mutual interactions between its constituents. When the mean free path of a parton becomes too large, the description in terms of partonic degrees of freedom ceases to be valid; one is instead left with an expanding gas of hadrons. Whether the dense parton gas reaches a state of kinetic equilibrium before it hadronizes is a question of great interest for the interpretation of the data at RHIC and LHC. So far, most of the calculations concerning experimental signatures of the QGP rely on the assumption that the system is at least kinetically equilibrated.

It was commonly assumed for a long time (see e.g. [3]) that shortly after the nuclei collide, kinetic equilibrium is rapidly achieved on time scales $\lesssim 1 \mathrm{fm}$ (see e.g. 任). It was however shown that, due to the effect of expansion, at least elastic collisions may not be effective enough [5]. An important question is also to characterize the initial state of the parton system formed just after the collision [2,7]. The purpose of this paper is to reexamine the role of elastic collisions in the process of kinetic equilibration, assuming that, already at early times, a local Boltzmann equation may be written for the partonic phase space distribution. We specifically compare within a simple relaxation time approximation the case where initial conditions are given by the saturation scenario, which may already be valid at RHIC, to the case where the initial gluons are produced incoherently in the perturbative QCD regime, the so-called minijet scenario. We consider only the pure gluon case and $2 \rightarrow 2$ elastic collisions in the limit of small-angle scatterings. By calculating the time-dependent relaxation time in a self consistent way, we are able to obtain various observables as functions of time, thus probing kinetic equilibration. The method is described in sections $\amalg$ to IV. In section $\mathrm{V}$, we present our results. Comparing with Ref. [8], where the exact solution is worked out in the saturation scenario, one can assess the validity of our approach, which appears to be surprisingly good. We then apply this method to the minijet scenario, considered in Ref. [9], and we consistently take care of conservation laws. We argue that, to characterize equilibration, a reliable criterium is to test the isotropy of various observables. As a consequence we find in particular that for both initial conditions the system does not reach kinetic equilibrium at RHIC energies. We discuss the reliability of our description and argue in particular that, due to the fragility of the weak coupling approximation, it appears difficult to obtain definite conclusions at LHC energies. In any case, the kinetic equilibration time is an order of magnitude bigger than the typical $1 \mathrm{fm}$ estimate usually assumed. The hypothesis that elastic collisions are sufficient to rapidly achieve kinetic equilibration, often made in the literature [3] is thus shown to be wrong, as already suggested in [5]. Similar conclusions have been obtained in a slightly different context by the authors of Ref. 10]. 


\section{THE BOLTZMANN EQUATION IN THE SMALL ANGLE APPROXIMATION}

Shortly after the gluons have been produced, the occupation number becomes small enough so that one can treat the further evolution by means of a Boltzmann equation describing the time evolution of the local distribution $f(\vec{p}, \vec{x}, t)$ for on-shell particles 1 . When the occupation number becomes smaller than unity, one can make the further approximation of a gas of classical particles. Here, we consider only the $g g \rightarrow g g$ elastic processes in the collision term, which we shall treat in the leading small-angle scattering approximation. Assuming one-dimensional expansion in the early stages after the collision and longitudinal boost invariance in the central region in the center of mass frame, the Boltzmann equation can be written in terms of a derivative at constant $p_{z} t[12]$ :

$$
\left.\partial_{t} f(\vec{p}, t)\right|_{p_{z} t}=\mathcal{C}(\vec{p}, t)
$$

Defining the moments $N_{s}=\left\langle p^{s}\right\rangle=\int_{\vec{p}} p^{s} f(\vec{p}, t)$, with the notation $\int_{\vec{p}} \equiv 2\left(N_{c}^{2}-1\right) \int d^{3} p /(2 \pi)^{3}$, one obtains for the collision term $\mathcal{C}$, in the limit of small-angle elastic scatterings between classical particles $(f \ll 1)$, to logarithmic accuracy [2,13,

$$
\mathcal{C}(\vec{p}, t)=\mathcal{L} N_{0} \nabla_{p}^{2} f(\vec{p}, t)+2 \mathcal{L} N_{-1} \vec{\nabla}_{p}[\vec{v} \cdot f(\vec{p}, t)]
$$

where $\vec{v}=\vec{p} / p$, and where $\mathcal{L}=\pi \alpha_{S}^{2} \frac{N_{c}^{2}}{N_{c}^{2}-1} \ln \left(1 / \chi_{\text {min }}^{2}\right)$, $\chi_{\text {min }}$ being the minimum scattering angle, whose value is determined by the physics of Debye screening. We shall come back to this later.

In the present paper, we will solve the Boltzmann equation in a relaxation time approximation, that is we will solve the equations for moments of the distribution $f$, taking into account however the microscopic dynamical information contained in (2.2).

\section{THE "SELF-CONSISTENT" RELAXATION TIME APPROXIMATION}

The Relaxation Time Approximation (RTA) simply consists in replacing the effect of the collisions in the Boltzmann equation (2.1) by an exponential-like relaxation towards a local "equilibrium" distribution'? $\mathcal{C} \equiv$

\footnotetext{
${ }^{1}$ One can argue that the requirement for using a local Boltzmann equation for on-shell quanta is that the occupation number $f$ be $\lesssim 1 / \alpha_{S}$, where $\alpha_{S}$ is the strong coupling constant [1].

${ }^{2}$ The distribution $f_{e q}$ has the meaning of a local equilibrium distribution only when the system has reached the hydrodynamic regime, where $\lambda(t)$ and $t^{1 / 3} T(t)$ are constants 14 .
}

$-\left(f-f_{e q}\right) / \theta$, with $f_{e q}(\vec{p}, t)=\lambda(t) \exp (-p / T(t))$. We assume that the relaxation time $\theta$ does not depend on $\vec{p}$. This has to be viewed as a mean-field like approximation : the different modes are effectively decoupled and their evolution is governed by an effective relaxation time which contains the microscopic information and is to be computed self-consistently. Indeed, strictly speaking this equation has no solution and one has to take it in a weaker sense, that is at the level of moments of the partonic distribution:

$$
\int_{\vec{p}} m(\vec{p}, t) \mathcal{C}(\vec{p}, t)=-\frac{\langle m\rangle(t)-\langle m\rangle_{e q}(t)}{\theta_{m}(t)},
$$

where $m(\vec{p}, t)$ is an arbitrary function, $\langle m\rangle_{(e q)}=$ $\int_{\vec{p}} m(\vec{p}, t) f_{(e q)}(\vec{p}, t)$, and $\theta_{m}(t)$ is the associated relaxation time, different choices for the function $m$ leading to different relaxation times.

Let us be more precise on this last point: it is clear that the RTA, being a one-time-scale ansatz, cannot fully reproduce the evolution of the whole ensemble of modes. A particular choice for the function $m$ picks up a particular momentum scale in the momentum distribution $f$, whose contribution to the moment $\langle m\rangle$ dominates. The relaxation toward isotropy of this part of the distribution is characterized by the time scale $\theta_{m}$. So the appropriate choice for the moment function $m$ depends on the momentum scale characterizing the physics one wants to study.

In this paper we are concerned with the question of kinetic equilibration, that is with the isotropy of "thermodynamic" quantities, like longitudinal and transverse pressures $P_{L}(t)=\left\langle p_{z}^{2} / p\right\rangle$ and $P_{T}(t)=\left\langle p_{\perp}^{2} / p\right\rangle$, where $p_{\perp}^{2}=\left(p_{x}^{2}+p_{y}^{2}\right) / 2$. The particles we are interested in have energy of the order of the average energy per particle $\bar{\epsilon}(t)=\epsilon(t) / n(t), \quad\left(n=N_{0}\right.$ and $\epsilon=N_{1}$ are the particle number and energy densities per unit volume respectively). A pertinent choice leading to simple equations is $m(\vec{p})=\left(p_{z}^{2}-p_{\perp}^{2}\right) / p$ or equivalently, $\langle m\rangle=P_{L}-P_{T}$. Inserting the Landau-Mueller collision term (2.2) in (3.1) and introducing the notation: $N_{s}^{z}=\left\langle p_{z}^{2} p^{s-2}\right\rangle$ and $N_{s}^{\perp}=\left\langle p_{\perp}^{2} p^{s-2}\right\rangle$, one obtains the following equation for the relaxation time:

$$
\frac{N_{1}^{z}-N_{1}^{\perp}}{\theta}=4 \mathcal{L} N_{0}\left(N_{-1}^{z}-N_{-1}^{\perp}\right)+2 \mathcal{L} N_{-1}\left(N_{0}^{z}-N_{0}^{\perp}\right) .
$$

The two other parameters of the relaxation time ansatz, $\lambda(t)$ and $T(t)$, are computed using conservation

\footnotetext{
${ }^{3}$ The choices $\langle m\rangle=P_{L}$ or $\langle m\rangle=P_{T}$ give the same results as those presented below. The choice $\langle m\rangle=\epsilon$ leads to the equation of energy conservation (see Eq. (3.3)) which contains no information about collisions.

${ }^{4}$ For example, $P_{L}=N_{1}^{z}, P_{T}=N_{1}^{\perp}$.
} 
laws: conservation of energy, and of particle number in elastic collisions 5 . In the RTA, these give the relations

$$
\begin{aligned}
& \epsilon(t)=\epsilon_{e q}(t)=6 \frac{N_{c}^{2}-1}{\pi^{2}} \lambda(t) T^{4}(t), \\
& n(t)=n_{e q}(t)=2 \frac{N_{c}^{2}-1}{\pi^{2}} \lambda(t) T^{3}(t) .
\end{aligned}
$$

Note that the particle number conservation law implies that the number density exactly falls like $t^{-1}: t n(t)=$ $t_{0} n\left(t_{0}\right)$, with $t_{0}$ the time when our description begins.

\section{SOLVING THE EQUATIONS}

The solution of the Boltzmann equation in the RTA is given by [12]

$$
\begin{aligned}
f\left(\vec{p}_{t}, p_{z}, t\right)= & f_{0}\left(\vec{p}_{t}, p_{z} \frac{t}{t_{0}}\right) \mathrm{e}^{-x(t)} \\
& +\int_{t_{0}}^{t} d t^{\prime} \frac{\mathrm{e}^{x\left(t^{\prime}\right)-x(t)}}{\theta\left(t^{\prime}\right)} f_{e q}\left(\vec{p}_{t}, p_{z} \frac{t}{t^{\prime}}, t^{\prime}\right),
\end{aligned}
$$

where $x(t)=\int_{t_{0}}^{t} d t^{\prime} \theta^{-1}\left(t^{\prime}\right), f_{0}(\vec{p})$ is the initial distribution, and where $\lambda(t), T(t)$ and $\theta^{-1}(t)$ are computed at each time with the help of Eqs. (3.2)-(3.4). We can use Eq. (4.1) to give an integral expression for all the quantities appearing in these equations. In the present case, every phase space integral can be computed analytically, thus considerably simplifying the numerical task. One typically obtains a formula of the type

$$
\begin{aligned}
M(t)= & M\left(t_{0}\right) \mathcal{F}_{M}^{(0)}\left(t_{0} / t\right) \mathrm{e}^{-x} \\
& +\int_{t_{0}}^{t} d t^{\prime} \frac{\mathrm{e}^{x^{\prime}-x}}{\theta^{\prime}} \mathcal{F}_{M}^{(e q)}\left(t^{\prime} / t\right) M_{e q}\left(t^{\prime}\right),
\end{aligned}
$$

where $x \equiv x(t), x^{\prime} \equiv x\left(t^{\prime}\right), \theta^{\prime} \equiv \theta\left(t^{\prime}\right)$ and $M \equiv N_{s}, N_{s}^{z}$ or $N_{s}^{\perp}(s=-1,0,1)$, and where the corresponding functions $\mathcal{F}_{M}^{(0)}$ and $\mathcal{F}_{M}^{(e q)}$ are calculable. The function $\mathcal{F}_{M}^{(0)}$ depends on the form of the initial distribution. The "equilibrium" moments $M_{e q}$, computed with $f_{e q}$, only depend on $\lambda$ and $T$ (see Eqs. A1 and (A2) in the appendix).

We solve the system of equations (3.2)-(3.4) and (4.2) by using a method similar to that described in Ref. [9]: suppose we know the parameters $\lambda, T$ and $\theta^{-1}$ for every time $t \leq t_{1}$. We want to compute their values at time $t_{1}+\delta t$. We use a first guess (for example the values at time $t_{1}$ ) to compute a first estimation $M^{(1)}$ of the moments $M\left(t_{1}+\delta t\right)$, using Eq. (4.2). We then compute a second estimation of our three parameters with the help of Eqs. (3.2)-(3.4), with which we obtain

\footnotetext{
${ }^{5}$ The "fugacity" parameter $\lambda(t)$ is needed to enforce particle number conservation in elastic collisions.
}

a second estimation $M^{(2)}$ of $M\left(t_{1}+\delta t\right)$, again using Eq. (4.2). We repeat these steps until convergence of our three parameters to some fixed accuracy. We have checked the validity of this method by solving Eq. (4.2) in some exactly solvable cases, for example by computing $n=N_{0}$ at each time-step with the help of Eq. (4.2), with $\mathcal{F}_{n}^{(0)}(a)=\mathcal{F}_{n}^{(e q)}(a)=a$.

\section{INITIAL CONDITIONS AND RESULTS}

In this section, we apply the above formalism to the study of kinetic equilibration of the gluon gas formed during the very early stages of the collision. We study two different scenarios for the initial condition, namely the saturation and minijet scenarios. The corresponding initial distributions are taken from Ref. [8] for the former case (see also [2]) and from Ref. [9] for the later.

To proceed further, one has to give a prescription for regularizing the logarithmic singularity $\mathcal{L}$ appearing in the collision term (Eq. (2.2)), in the small angle approximation.

\section{A. Screening}

The logarithmically divergent integral appearing in the small scattering angle collision integral is physically regulated by screening effects in the gluon medium [8,13,15]. The minimum scattering angle is given by the relation $m_{D}^{2}=\underline{p}^{2} \chi_{m i n}^{2}$, where $m_{D}$ is the Debye mass, an expression of which can be obtained in terms of the distribution function in the linear response approximation [15], and where $p$ is the typical momentum of particles in the medium. We shall see below that for early times, because of the expansion, the particles in the central region have essentially zero longitudinal momentum [ In this situation, the exchanged gluon is essentially transverse and the relevant screening mass is the transverse mass [8]

$$
m_{T}^{2}(t)=\frac{\alpha_{S} N_{c}}{\pi^{2}} \int \frac{d^{3} p}{p} f(\vec{p}, t)=\frac{4 \pi \alpha_{S} N_{c}}{N_{c}^{2}-1} N_{-1}(t),
$$

and we write $L \equiv \ln \left(1 / \underline{\chi_{\min }^{2}}\right)=\ln \left(\overline{\left\langle p_{t}^{2}\right\rangle} / m_{T}^{2}\right)$, where $p_{t}^{2}=p_{x}^{2}+p_{y}^{2}$ and where $\overline{\langle\ldots\rangle}=\langle\ldots\rangle / n$ denotes the average per particle. Although the above choice for $L$ is motivated by the highly anisotropic form of the distribution at early times, we shall use it also when the sys-

\footnotetext{
${ }^{6}$ This is explicit in the initial distribution corresponding to the saturation scenario (see Eq. (5.1)). In the minijet scenario, although the initial distribution is isotropic in momentum space, it becomes rapidly peaked around $p_{z}=0$ (see Fig. (4).
} 
tem approaches kinetic equilibrium 7 . It should be noted here that the validity of the logarithmic approximation is marginal because of the fact that the coupling constant is not very small compared to one (in particular, one has typically $\alpha_{S} N_{c} \simeq 1$, see below) 8 .

\section{B. Saturation scenario}

In the saturation scenario one assumes that the produced transverse energy per unit rapidity is dominated by small-x gluons having a transverse momentum $p_{t} \sim Q_{s}$, the saturation momentum [2]. These gluons are essentially freed during the collision, after a time $t_{i} \sim 1 / Q_{s}$ which is also the time needed for gluons in different units of rapidity to physically separate from each other. After a time $t_{i}$, gluons populating the central region in the center of mass frame of the collision have essentially no longitudinal momentum $\left(p_{z} t_{i} \sim p_{z} / Q_{s} \ll 1\right)$. Following Mueller [2], one writes for the occupation number in the central region at $t_{i}$ :

$$
f_{s a t}\left(\vec{p}, t_{i}\right)=\frac{c}{\alpha_{S} N_{c}} \delta\left(p_{z} t_{i}\right) \Theta\left(Q_{s}^{2}-p_{t}^{2}\right),
$$

where $c \sim 1$ is a numerical constant, which has been computed numerically using a classical field approximation in Ref. 117. For an $S U(2)$ gauge theory, $c=1.3$. The approximation of classical particles makes sense when the occupation number $f \lesssim 1$ so that, following the authors of Ref [8], we take, as initial time, $t_{0}=\frac{c}{\alpha_{S} N_{c}} t_{i}$ where one has, assuming free streaming between $t_{i}$ and $t_{0}$,

$$
f_{0}(\vec{p})=f_{\text {sat }}\left(\vec{p}, t_{0}\right)=\delta\left(p_{z} t_{i}\right) \Theta\left(Q_{s}^{2}-p_{t}^{2}\right)
$$

The values of the parameters corresponding to RHIC and LHC energies given in [8] are summarized in Tab. If In the following we take $\alpha_{S}=0.3, N_{c}=3$. We evolve the system until the particle number density becomes lower than 1 per $\mathrm{fm}^{3}$, when the description in terms of partonic degrees of freedom becomes meaningless. The corresponding time $t_{\max }$ is evaluated using the conservation of the particle number: $t n(t)=t_{0} n\left(t_{0}\right)$ (see Tab. II). This rough estimate serves only to give an upper limit for our description. In particular one obtains $t_{\max } \simeq 30 \mathrm{fm}$ at LHC, which is too long for the approximation of longitudinally boost invariant geometry to be valid. Nevertheless, we present our results for $t \leq t_{\max }$ in order to show the behavior of the system.

Fig. 1 shows the logarithm $L$ as a function of time. At early times, $L \ll 1$ whatever the way one chooses

\footnotetext{
${ }^{7}$ For an isotropic distribution, a more appropriate choice would be $L_{i s o}=\ln \left(\overline{\left\langle p^{2}\right\rangle} / m_{D}^{2}\right)$. In this case however $m_{D}^{2}=$ $2 m_{T}^{2},\left\langle p_{t}^{2}\right\rangle=2\left\langle p^{2}\right\rangle / 3$, and $L-L_{\text {iso }}=\ln 4 / 3 \simeq 0.3$.

${ }^{8}$ For more details on this point, see [16].
}

to implement screening. As time goes on, although the situation becomes better, the logarithmic approximation remains marginal.

We first test our relaxation time approximation by comparing our results with those of Ref. [8]. We show in Fig. 2 the time evolution of the energy density $\epsilon$ and the longitudinal and transverse pressures $P_{L}$ and $P_{T}$ at RHIC, as well as those of the mean longitudinal and transverse squared momentum per particle $\overline{\left\langle p_{z}^{2}\right\rangle}$ and $\overline{\left\langle p_{\perp}^{2}\right\rangle}$, both for RHIC and LHC energies. These are to be compared with Figs. 7 and 9 of [8] (we choose the units so as to make the comparison easiest): one observes a semiquantitative agreement with the exact solution, which is remarkably good in view of the simplicity of our approach.

We now come to the study of kinetic equilibration of the gluon gas. To this end, we measure the anisotropy of the distribution by means of the ratios

$$
R_{k}=\frac{\left\langle p_{z}^{2} / p^{k}\right\rangle}{\left\langle p_{\perp}^{2} / p^{k}\right.}=\frac{N_{z}^{k}}{N_{\perp}^{k}},
$$

which should approach 1 as the system approaches kinetic equilibrium. We stress here that the criterium we use to characterize kinetic equilibrium (the isotropy of the distribution) is quite different from that used in [8]. As a consequence, although we have essentially similar results, we arrive at quite different conclusions. Indeed, the time evolution of $R_{k}$ for $k=0, \ldots, 3$, at RHIC and LHC energies, is shown in Fig. 3, where one can see that the system is far from being isotropic even at $t \sim 10 \mathrm{fm}$ at RHIC. The situation looks better at LHC, where $R_{k} \simeq 0.8$ for $t \simeq 10 \mathrm{fm}$. It is to be noted that in this scenario, the only scale is $Q_{s}$ and consequently the results for RHIC and LHC are essentially the same when everything is expressed in units of $Q_{s}$.

\section{Minijet scenario}

In the minijet scenario, the initial gluons are produced by means of hard and semi-hard collisions between the partons of the incident nuclei, and one can compute the initial energy and particle number density in perturbative QCD [1]. The initial gluon multiplicity is dominated by the softest of these hard gluons, having an energy $p_{0} \sim$ $1-2 \mathrm{GeV}$, which are produced on a typical time $t_{0} \sim$ $1 / p_{0}$. Following the author of Ref. [9], we parametrize the initial state by a Boltzmann distribution. In the central region, we have

$$
f_{0}(\vec{p})=f_{j e t}\left(\vec{p}, t_{0}=1 / p_{0}\right)=\lambda_{j e t} \mathrm{e}^{-p / T_{j e t}},
$$

where the parameters $\lambda_{j e t}=\lambda\left(t_{0}\right)$ and $T_{j e t}=T\left(t_{0}\right)$ are determined from the initial energy and particle number densities: $\epsilon_{\text {jet }}=\epsilon\left(t_{0}\right)=\epsilon_{e q}\left(t_{0}\right)$ and $n_{\text {jet }}=n\left(t_{0}\right)=$ $n_{e q}\left(t_{0}\right)$. We recall the values of the parameters used in 9 for RHIC and LHC in Tab. II. As in the previous section, we use $\alpha_{S}=0.3, N_{c}=3$. Concerning the validity 
of the logarithmic approximation, the situation is more confortable here: in the time interval we considered, we have $1.5 \lesssim L \lesssim 5$.

Fig. 4 shows the time evolution of the ratio $R_{k}$ defined in (5.2) for both RHIC and LHC energies. As expected, one observes a regime a free streaming at very early times, until the effect of collisions begins to set in, that is for time $t-t_{0} \ll \theta\left(t_{0}\right)$. Concerning the degree of isotropy the system reaches, the situation is even worth than in the saturation scenario: the system is always far from isotropy at RHIC; at LHC, the system is still quite anisotropic $\left(R_{k} \lesssim 0.8\right)$ until $t \sim 30 \mathrm{fm}$. Here again, we note that the only scale of the description is $T_{j e t}$, and the results for RHIC and LHC are essentially the same when expressed in units of $T_{\text {jet }}$.

The above conclusions are to be compared with those of Ref. [9], where the authors obtain relatively short "equilibration times", of the order of $4-5 \mathrm{fm}$, both at RHIC and LHC. However, although only elastic collisions between gluons are considered, the total number of particles is not conserved in [9], and in fact grows with time (the number density $n$ falls slower than $1 / t$ ). This means in particular that collisions between particles are anomalously frequent and consequently the system equilibrates anomalously fast. The similarity of equilibration times obtained in [9] at RHIC and LHC is due to the fact that the authors work with a running coupling constant which grows with time, as the typical momentum of the particles decreases. The case of a running coupling constant is examined in next section. We shall see that our previous conclusions remain unchanged.

\section{Discussion}

Let us first remark that the model used here to describe the evolution of the gluon gas can only give qualitative information. Here we use it to study the question: are the elastic collisions efficient enough to achieve kinetic equilibrium, and if so on which time scale? In order to obtain a reliable answer, let us study the sensitivity of the above results to the details of the description, for example the value of $\alpha_{S}$ or the prescription used to cutoff the logarithmic divergence in the small-angle limit.

\section{Running coupling constant}

It is interesting to consider the case where the coupling constant is allowed to run, growing with time as the typical energy of the particles in the medium decreases. As mentionned above, this should accelerate the equilibration process. Here we shall compute the coupling constant at each time as a function of the mean energy per particle: $\alpha_{S}(t) \equiv \alpha_{S}(\mu=\bar{\epsilon}=3 T)$, with

$$
\alpha_{S}(\mu)=\alpha_{S}\left(M_{Z}\right) \frac{\ln \left(M_{Z} / \Lambda_{Q C D}\right)}{\ln \left(\mu / \Lambda_{Q C D}\right)}
$$

where $M_{Z} \simeq 90 \mathrm{GeV}$ the mass of the $Z^{0}$ boson, $\alpha_{S}\left(M_{Z}\right)=0.1$, and where we take $\Lambda_{Q C D} \simeq 200 \mathrm{MeV}$.

In the minijet scenario, at LHC, we have $0.2<\alpha_{S}(t)<$ 0.4 on the time interval we considered, and we obtain very similar results to the case where $\alpha_{S}=0.3$. At RHIC, $0.3<\alpha_{S}(t)<0.5$ and, although the situation is better when compared to the fixed $\alpha_{S}$ case, the system still does not reach kinetic equilibrium. The corresponding curves for the ratio $R_{1}=P_{L} / P_{T}$ are shown in Fig. 5.

In the saturation scenario, the mean energy per particle is smaller than in the previous case and the coupling constant becomes rapidly $\gtrsim 0.5$. and the results have no physical significance. This is to be viewed as a manifestation of the fragility of the weak coupling approximation.

\section{Robustness of the results}

In order to estimate roughly the accuracy of our results, we give two estimates, simply replacing $L \rightarrow 2 L$ and $L \rightarrow L / 2$ in the collision term, keeping $\alpha_{S}=0.3$. The corresponding curves for the ratio $R_{1}=P_{L} / P_{T}$ are shown in Figs. 5 for both scenarios, at RHIC and LHC energies. In the minijet scenario we also show the curves corresponding to the case where the coupling constant depends on time, which are seen to be contained within our "uncertainty bands". One observes that the qualitative conclusions of previous sections are unchanged: in both scenarios, elastic collisions are not sufficient to drive the system towards kinetic equilibrium at RHIC energies. At LHC, kinetic equilibrium may be reached in the saturation scenario even for times reasonnably short for the approximation of longitudinal expansion to have a sense, say $t \sim 10 \mathrm{fm}$. However, it appears difficult to draw any firm conclusion because of the uncertainties of our qualitative description. In the minijet scenario at LHC energy the situation is even worst and one cannot draw definite conclusions concerning kinetic equilibration even for very long times. What one can say however is that, in both scenarios, the assumption that kinetic equilibration is rapidly achieved by means of elastic collisions only, on time scales $\lesssim 1 \mathrm{fm}$, is not reliable at RHIC energy and questionnable at LHC energy, where the actual equilibration time is at least of the order of a few fermis.

\section{CONCLUSION}

We have studied the kinetic equilibration of the gluon gas initially produced in very high energy nuclear collisions, by considering $2 \rightarrow 2$ small angle elastic scatterings only. Two different scenarios have been assumed for the production of such gluons: the saturation and the minijet scenarios. By using a simple "self-consistent" relaxation time approximation, we are able to reproduce semi-quantitative features of the exact solution of the Boltzmann equation. By measuring the anisotropy of 
different observables as a function of time, we can follow the system toward kinetic equilibrium. Our results show that elastic collisions are not as efficient as usually believed to achieve kinetic equilibrium. This contradicts a widely used assumption.

This does not means that kinetic equilibrium is not achieved in a real collision. Indeed, concerning the physical question of kinetic equilibration, the present study is only preliminary as one should include other contributions in the collision integral. In particular, inelastic branching processes have recently been proposed as playing a very important role in this respect [6].

\section{ACKNOWLEDGMENTS}

We acknowledge very useful discussions with R. Baier, A. Krzywicki and A. H. Mueller. LPT is laboratoire associ au CNRS - URA00063.

\section{APPENDIX: MOMENTS OF THE DISTRIBUTION}

We give here the explicit form of the equilibrium moments $M_{e q}$ and of the functions $\mathcal{F}_{M}^{(0, e q)}$ appearing in Eq. (4.2), for the different moments $M$ used in this paper. These are of three different sort: "isotropic", as $N_{s}=\left\langle p^{s}\right\rangle$, "longitudinal", as $N_{s}^{z}=\left\langle p_{z}^{2} p^{s-2}\right\rangle$, and "transverse", $N_{\perp}^{z}=\left\langle p_{\perp}^{2} p^{s-2}\right\rangle$, with the obvious relation: $N_{s}=N_{s}^{z}+2 N_{s}^{\perp}$. Introducing the notation

$$
\{A \| B\}=\left\{\begin{array}{l}
A \text { if } f_{0}(\vec{p})=f_{e q}\left(\vec{p}, t_{0}\right) \\
B \text { if } f_{0}(\vec{p})=\delta\left(p_{z}\right) g\left(p_{\perp}\right),
\end{array}\right.
$$

one easily gets, using Eq. (4.1),

$$
\begin{aligned}
t \mathrm{e}^{x} N_{s}(t)= & t_{0} N_{s}\left(t_{0}\right)\left\{h_{s}\left(t_{0} / t\right) \| 1\right\} \\
& +\int_{t_{0}}^{t} \frac{d t^{\prime}}{\theta^{\prime}} t^{\prime} \mathrm{e}^{x^{\prime}} N_{s}^{e q}\left(t^{\prime}\right) h_{s}\left(t^{\prime} / t\right),
\end{aligned}
$$

and

$$
\begin{aligned}
t^{3} \mathrm{e}^{x} N_{s}^{z}(t)= & t_{0}^{3} N_{s}\left(t_{0}\right)\left\{h_{s}^{z}\left(t_{0} / t\right) \| 0\right\} \\
& +\int_{t_{0}}^{t} \frac{d t^{\prime}}{\theta^{\prime}}\left(t^{\prime}\right)^{3} \mathrm{e}^{x^{\prime}} N_{s}^{e q}\left(t^{\prime}\right) h_{s}^{z}\left(t^{\prime} / t\right)
\end{aligned}
$$

where

$$
N_{s}^{e q}(t)=\int_{\vec{p}} p^{s} f_{e q}(\vec{p}, t)=(s+2) ! \frac{N_{c}^{2}-1}{\pi^{2}} \lambda(t) T^{s+3}(t),
$$

and where

$$
\begin{aligned}
& h_{s}(a)=\int_{0}^{1} d x\left[1-\left(1-a^{2}\right) x^{2}\right]^{s / 2}, \\
& h_{s}^{z}(a)=\int_{0}^{1} d x x^{2}\left[1-\left(1-a^{2}\right) x^{2}\right]^{s / 2-1} .
\end{aligned}
$$

Eqs. (A1)-A2 can be written in the generic form of Eq. 4.2), using the following definitions: $\mathcal{F}_{N_{s}}^{e q}(a)=$ $a h_{s}(a)$ and $\mathcal{F}_{N_{s}^{z}}^{e q}(a)=3 a^{3} h_{s}^{z}(a)$.

Writing $A=\sqrt{1-a^{2}}$, one obtains:

$$
\begin{aligned}
& h_{-1}(a)=\frac{\arcsin A}{A}, h_{1}(a)=\frac{a+h_{-1}(a)}{2}, \\
& h_{-2}(a)=\frac{\arctan A}{A}, h_{2}(a)=\frac{2+a^{2}}{3} .
\end{aligned}
$$

The functions $h_{s}^{z}$ can be deduced from the previous ones by using the obvious relation

$$
h_{s}^{z}(a)=\frac{2}{s} \frac{d}{d a^{2}} h_{s}(a) .
$$

For the particular case $s=0$, one obtains

$$
h_{0}(a)=1, h_{0}^{z}(a)=\frac{1}{A^{2}}\left(\frac{\arctan A}{A}-1\right) .
$$

[1] K. Kajantie, P.V. Landshoff and J. Lindfors, Phys. Rev. Lett. 59 (1987) 2527; J.P. Blaizot and A.H. Mueller, Nucl. Phys. B289 (1987) 847.

[2] A. H. Mueller, Nucl. Phys. B572 (2000) 227; Phys. Lett. B475 (2000) 220.

[3] T.S. Biró, E. van Doorn, B. Mller, M.H. Thoma and X.N. Wang, Phys. Rev. C 48 (1993) 1275 ; D.M. Elliott and D.H. Rischke, Nucl. Phys. A671 (2000) 583.

[4] G. Baym, H. Monien, C.J. Pethick and D.G. Ravenhall, Phys. Rev. Lett. 64 (1990) 1867.

[5] S.M.H. Wong, Phys. Rev. C 54 (1996) 2588.

[6] R. Baier, A. H. Mueller, D. Schiff and D. T. Son, Phys. Lett. B502 (2001) 51.

[7] H. Heiselberg and X. N. Wang, Phys. Rev. C 53 (1996) 1892, Nucl. Phys. B462 (1996) 389.

[8] J. Bjoraker and R. Venugopalan, Phys. Rev. C 63 (2001) 024609.

[9] G.C. Nayak, A. Dumitru, L. Mc Lerran, W. Greiner, Nucl. Phys. A687 (2001) 457.

[10] A. Dumitru and M. Gyulassy, Phys. Lett. B494 (2000) 215.

[11] A.H. Mueller, private communication.

[12] G. Baym, Phys. Lett. B138 (1984) 18.

[13] E.M. Lifshitz and L.P. Pitaevskii, Physical kinetics, Pergamon Press, 1981.

[14] J.D. Bjorken, Phys. Rev, D 27 (1983) 140.

[15] T.S. Biró, B. Müller and X.N. Wang, Phys. Lett. B283 (1992) 171.

[16] J. Serreau, 'Out-of-equilibrium phenomena in high energy nuclear collisions', PhD Thesis, LPT-ORSAY 01/27, hep-ph/0104023.

[17] A. Krasnitz and R. Venugopalan, Phys. Rev. Lett. 84 (2000) 4309; hep-ph/0007108. 


\begin{tabular}{c|ccccc}
\hline \hline SATURATION & $Q_{s}(\mathrm{GeV})$ & $t_{0}(\mathrm{fm})$ & $n\left(t_{0}\right)\left(\mathrm{fm}^{-3}\right)$ & $\epsilon\left(t_{0}\right)\left(\mathrm{GeV} / \mathrm{fm}^{3}\right)$ & $t_{\max }(\mathrm{fm})$ \\
\hline RHIC & 1. & 0.4 & 18.1 & 12.0 & $\sim 10$ \\
\hline LHC & 2. & 0.18 & 163.4 & 217.9 & $\sim 30$ \\
\hline
\end{tabular}

TABLE I. Values of the parameters $Q_{s}$ and $t_{0}$ characterizing the initial state in the saturation scenario at RHIC and LHC energies. The energy and particle number densities are computed from the initial distribution Eq. (5.1). The time $t_{\max }$ is the time after which the particle density becomes less than $1 / \mathrm{fm}^{3}$ and is evaluated using particle number conservation: $\operatorname{tn}(t)=t_{0} n\left(t_{0}\right)$.

\begin{tabular}{c|cccccc}
\hline \hline MINIJET & $t_{0}(\mathrm{fm})$ & $n_{\text {jet }}\left(\mathrm{fm}^{-3}\right)$ & $\epsilon_{\text {jet }}\left(\mathrm{GeV} / \mathrm{fm}^{3}\right)$ & $\lambda_{\text {jet }}$ & $T_{\text {jet }}(\mathrm{GeV})$ & $t_{\max }(\mathrm{fm})$ \\
\hline RHIC & 0.18 & 34.3 & 56.0 & 1.0 & 0.535 & $\sim 10$ \\
\hline LHC & 0.09 & 321.6 & 1110.0 & 1.0 & 1.13 & $\sim 30$ \\
\hline \hline
\end{tabular}

TABLE II. Values of the energy and particle number densities characterizing the state of the gluon system at the initial time $t_{0}$ in the minijet scenario, at RHIC and LHC energies. The values of the parameters $\lambda_{\text {iet }}$ and $T_{\text {jet }}$ characterizing the assumed initial distribution Eq. (5.3) are obtained from Eqs. (3.3)-(3.4) at $t=t_{0}$. The time $t_{\max }$ is the time after which the particle density becomes less than $1 / \mathrm{fm}^{3}$. 


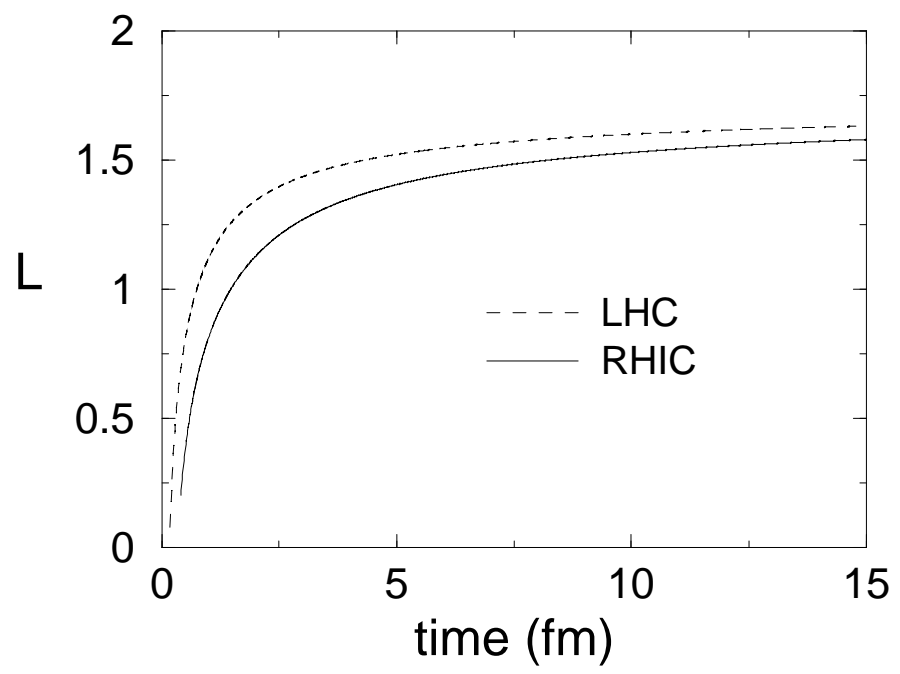

FIG. 1. The logarithm $L$ (see section $\mathrm{VA}$ ) as a function of time (in fm) in the saturation scenario at RHIC and LHC.

RHIC
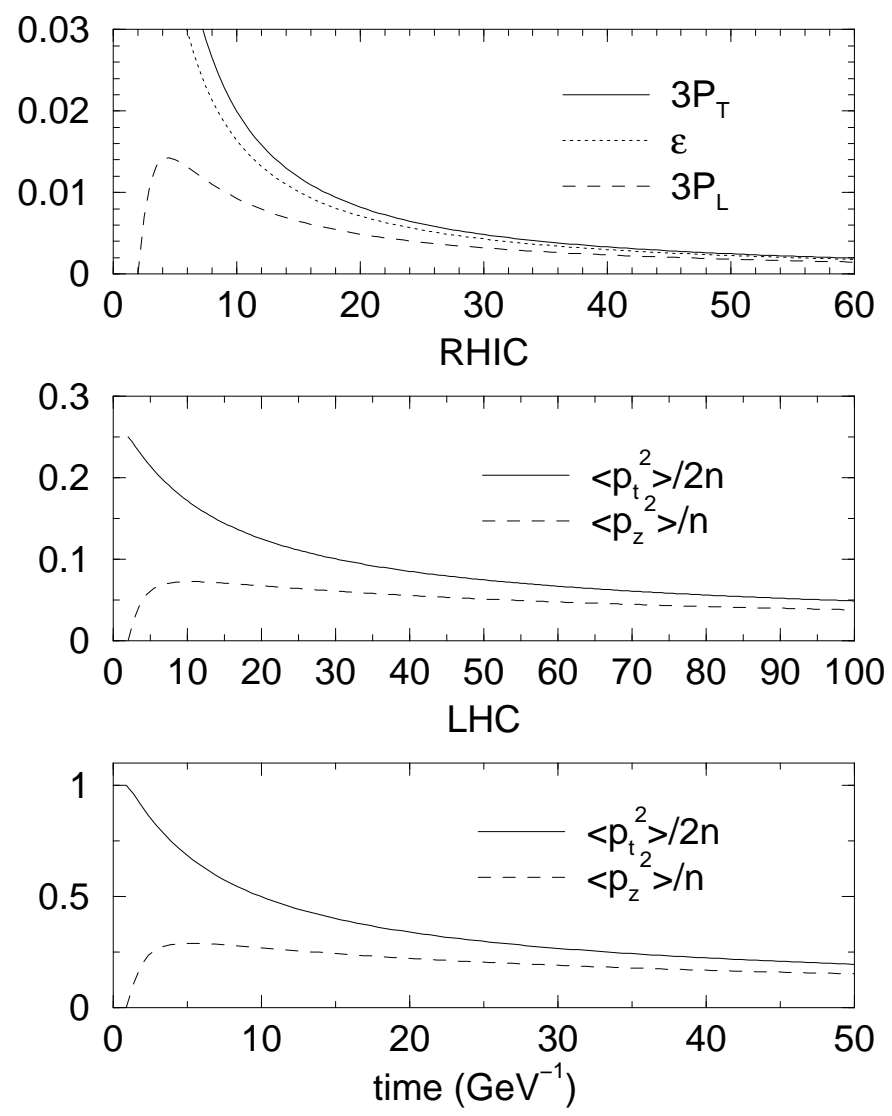

FIG. 2. Various observables as functions of time in the saturation scenario. The upper panel shows the time evolution of the energy density and of the longitudinal and transverse pressures (times 3 ) at RHIC. The second and third panels show the evolution of the average longitudinal and transverse squared momenta per particle $\overline{\left\langle p_{z}^{2}\right\rangle}$ and $\overline{\left\langle p_{\perp}^{2}\right\rangle}$ at RHIC and LHC respectively. Comparing with the curves obtained in [8], one observes a semi-quantitative agreement (in the above figure, everything is expressed in units of $\mathrm{GeV}$, as in [8]). 
RHIC

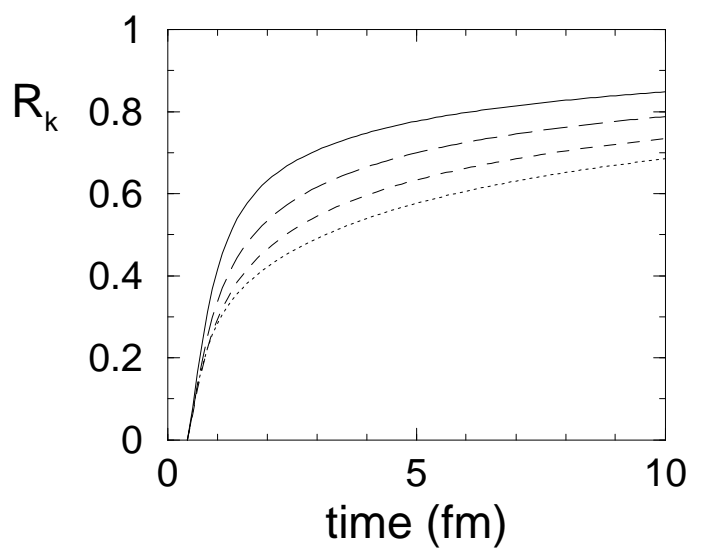

LHC

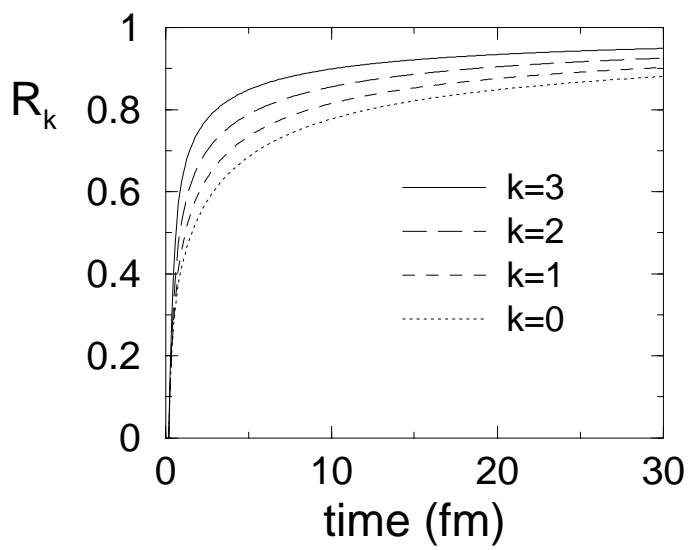

FIG. 3. Time evolution of the ratios $R_{k}$, defined in Eq. (5.2) in the saturation scenario at RHIC (left) and LHC (right). The quantities $1-R_{k}$ give a measure of the anisotropy of the microscopic distribution $f(\vec{p}, t)$ for different momentum scales, the larger $k$ the lower the probed momentum scale. These curves are obtained with $\alpha_{S}=0.3$ and the logarithm $L=\ln \left(\overline{\left\langle p_{t}^{2}\right\rangle} / m_{T}^{2}\right)$ (see section $\mathrm{VA}$.)
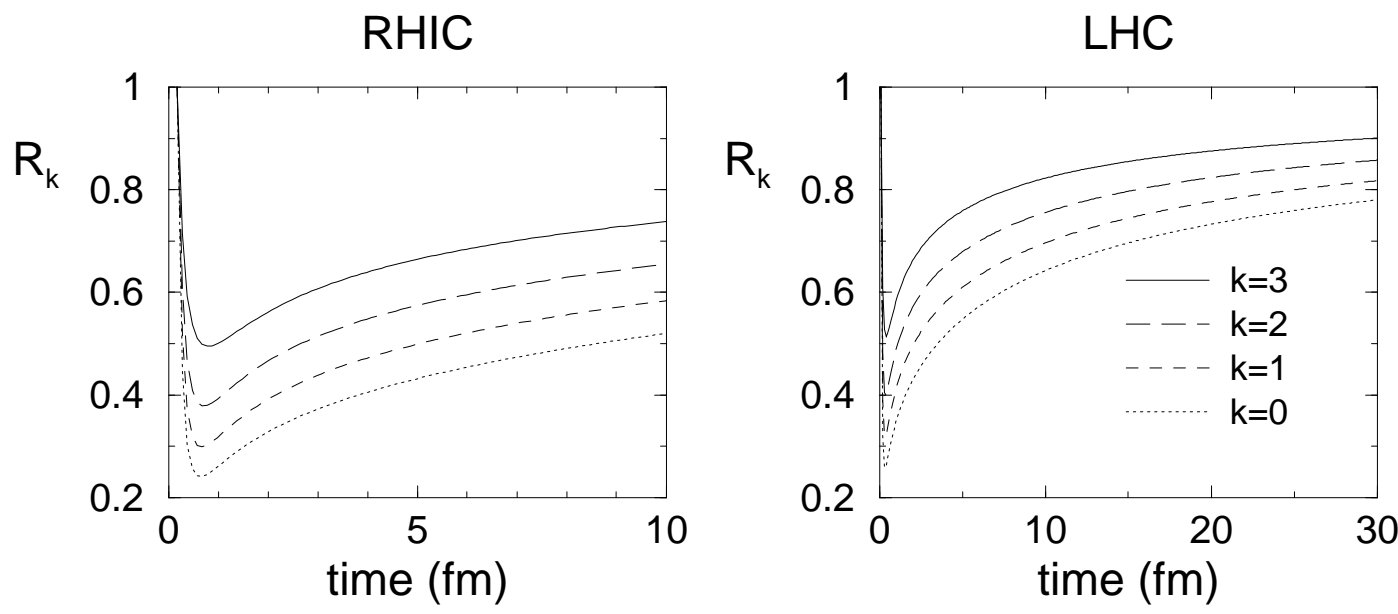

FIG. 4. Time evolution of the ratios $R_{k}$, defined in Eq. (5.2) in the minijet scenario at RHIC (left) and LHC (right). These curves are obtained with $\alpha_{S}=0.3$ and the logarithm $L=\ln \left(\overline{\left\langle p_{t}^{2}\right\rangle} / m_{T}^{2}\right)$ (see section $\mathrm{VA}$.) 
SATURATION : RHIC

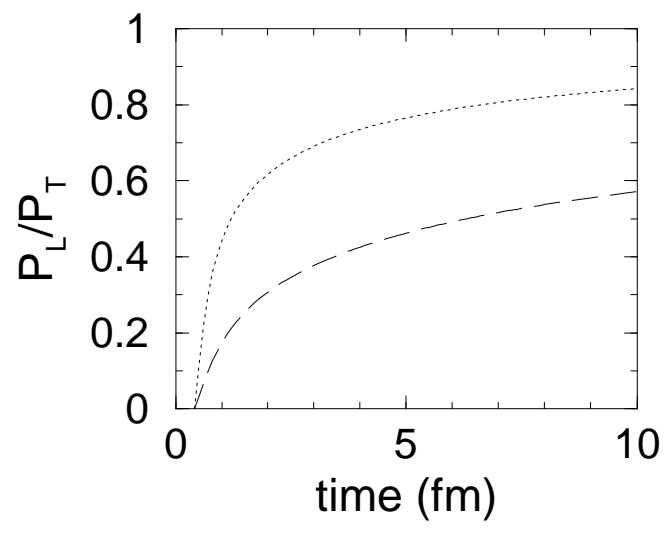

MINIJET : RHIC

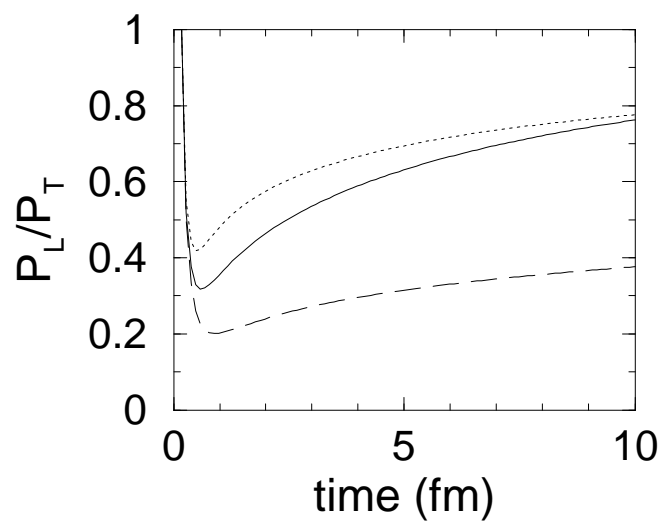

SATURATION : LHC
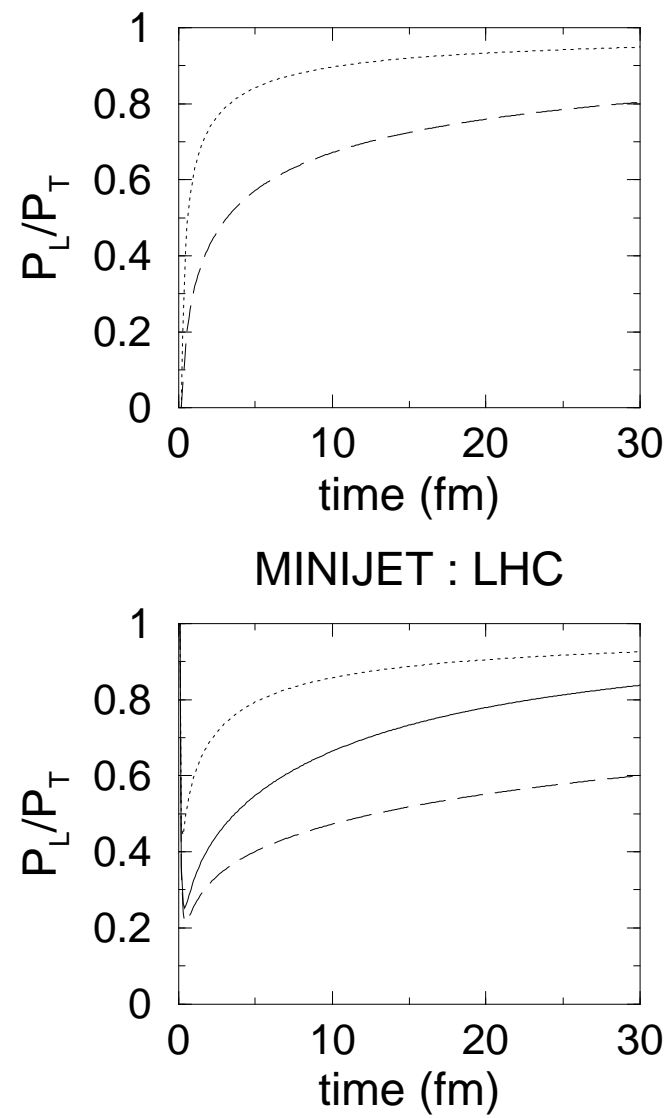

FIG. 5. Sensitivity of the results (here for the time evolution of the ratio $R_{1}=P_{L} / P_{T}$ ) with respect to the details of the description in the saturation (upper panels) and minijet (lower panels) scenarios at RHIC (left panels) and LHC (right panels) energies. Compared to the results shown in Figs. 3 and 4, the dotted curves are obtained by replacing the logarithm $L \rightarrow 2 L$ and the dashed curves by replacing $L \rightarrow L / 2$. The solid curves in the minijet scenario correspond to the case where the coupling constant runs with time (see Eq. (5.4)). 